\title{
Epsilon-hypercyclic operators
}

\author{
CATALIN BADEA $\dagger$, SOPHIE GRIVAUX $\dagger$ and VLADIMIR MÜLLER $\ddagger \dagger$ \\ † Laboratoire Paul Painlevé, UMR CNRS 8524, Université des Sciences et \\ Technologies de Lille, 59655 Villeneuve d'Ascq Cedex, France \\ $\ddagger$ Institute of Mathematics AV CR, Zitna 25, 11567 Prague 1, Czech Republic \\ (e-mail: \{badea,grivaux\}@math.univ-lille1.fr, muller@math.cas.cz)
}

(Received? July 2008)

\begin{abstract}
For each fixed number $\varepsilon$ in $(0,1)$ we construct a bounded linear operator on the Banach space $\ell_{1}$ having a certain orbit which intersects every cone of aperture $\varepsilon$, but with every orbit avoiding a certain ball of radius $d$, for every $d>0$. This answers a question from [8]. On the other hand, if $T$ is an operator on the Banach space $X$ such that for every $\varepsilon>0$ there is a point in $X$ whose orbit under the action of $T$ meets every cone of aperture $\varepsilon$, then $T$ has a dense orbit.
\end{abstract}

\section{Introduction}

The aim of this paper is to study some (variations of) density properties of orbits of bounded linear operators acting on a real or complex separable Banach space $X$. Using a Functional Analysis terminology, an operator $T \in \mathcal{B}(X)$ is said to be hypercyclic if there exists a vector $x \in X$ such that the orbit $\mathcal{O} r b(x, T)=$ $\left\{T^{n} x ; n \geq 0\right\}$ of $x$ under the action of $T$ is dense in $X$. A vector $x$ with dense orbit is called a hypercyclic vector for $T$.

While the first examples of Banach and Hilbert space hypercyclic operators are relatively recent $([\mathbf{1 0}])$, there is now an important literature on hypercyclicity properties and the dynamics of bounded linear operators. We refer the reader to the recent book [1] for more on this topic. It is natural in this context to investigate which properties of the orbit of a vector, weaker than denseness, imply either that the orbit itself is in fact dense, or that the operator is hypercyclic (i.e. some other orbit is dense in $X$ ). Let us mention here some of the results in this direction:

- if the orbit $\mathcal{O} \operatorname{rb}(x, T)$ is somewhere dense in $X$, then it is dense in $X[\mathbf{3}]$. This implies in particular that if the union of finitely many orbits $\mathcal{O} r b\left(x_{1}, T\right), \mathcal{O} r b\left(x_{2}, T\right)$,

$\dagger$ The first two authors were partially supported by ANR-Projet Blanc DYNOP. The third author was partially supported by grant No. 201/06/0128 of GA CR. All three authors were partially supported by the RiP-programme of the Mathematisches Forschungsinstitut Oberwolfach. We would like to thank the Institut for excellent working conditions. 
$\ldots, \mathcal{O} r b\left(x_{n}, T\right)$ is dense in $X$, then one of these orbits must already be dense. This result was proved directly in $[\mathbf{5}]$ and $[\mathbf{9}]$.

- suppose that for some positive number $d$ the orbit of $x \in X$ meets every open ball $B(y, d)$ of radius $d$. Then $\mathcal{O} r b(x, T)$ is not necessarily dense in $X$, but $T$ must be hypercyclic $[\mathbf{6}]$.

- if $x$ is a frequently hypercyclic vector for $T$, then $T \oplus T$ must be hypercyclic on $X \oplus X[7]$.

Recall that $x$ is a frequently hypercyclic vector for $T$ if for every non empty open subset $U$ of $X$, the set of positive integers $n$ such that $T^{n} x \in U$ has positive lower density; in other words, for every non empty open subset $U$ of $X$, there exists a sequence $\left(n_{k}\right)_{k \geq 0}$ with $n_{k}=O(k)$ such that $T^{n_{k}} x \in U$.

On the other hand, some conditions on the orbit, which may look strong enough at first sight, do not imply that the operator is hypercyclic. For instance:

- there exist operators which are weakly hypercyclic, i.e. for which there exists a vector $x$ whose orbit is weakly dense in $X$, but still are not hypercyclic: examples of weighted shifts having this property are given in [4].

- for every $\varepsilon>0$, there exists a bounded operator on the space $\ell_{1}(\mathbb{N})$ which has the following property: there exists a vector $x \in \ell_{1}(\mathbb{N})$ such that for every non empty open subset $U$ of $\ell_{1}(\mathbb{N})$, there exists a sequence $\left(n_{k}\right)_{k \geq 0}$ with $n_{k}=O\left(k^{1+\varepsilon}\right)$ such that $T^{n_{k}} x \in U$, but $T \oplus T$ is not hypercyclic [2]. This shows that the result of $[7]$ that every frequently hypercyclic operator on a Banach space satisfies the Hypercyclicity Criterion is in a sense optimal.

We investigate in this paper a weaker version of Feldman's result [6] already mentioned above: it states that if given a positive $\varepsilon$ there exists a vector $x$ such that for every $y \in X\left\|T^{n} x-y\right\| \leq \varepsilon$ for some integer $n$, then $T$ is hypercyclic.

Definition. Let $\varepsilon$ be a number in $(0,1)$. If $x$ is a vector of $X$, we say that $x$ is $\varepsilon$-hypercyclic if for every non zero vector $y \in X$ there exists an integer $n$ such that $\left\|T^{n} x-y\right\| \leq \varepsilon\|y\|$. The operator $T$ is $\varepsilon$-hypercyclic if it admits an $\varepsilon$-hypercyclic vector.

In particular, the orbit of $x$ must intersect every cone of a fixed aperture. This is in a sense a "scaled" version of the $\varepsilon$-density considered in Feldman's work. It is obviously weaker than Feldman's condition, and in a sense more natural in this context. The following question was proposed in [8]:

QUESTION 1.1. Suppose that $T$ is a bounded operator on $X$ which admits for some $\varepsilon \in(0,1)$ an $\varepsilon$-hypercyclic vector. Is it true that $T$ is hypercyclic?

The restriction $\varepsilon \in(0,1)$ comes from the fact the zero vector is trivially 1hypercyclic for any operator $T$.

The main result of this paper gives a negative answer to Question 1.1:

TheOREM 1.2. For every $\varepsilon \in(0,1)$ there exists an $\varepsilon$-hypercyclic operator on the space $\ell_{1}(\mathbb{N})$ which is not hypercyclic. 
Still:

THEOREM 1.3. If $T$ is a bounded operator on $X$ which is $\varepsilon$-hypercyclic for every $\varepsilon>0$, then $T$ must be hypercyclic.

We therefore obtain, together with Feldman's result, the geometric statement stated in the abstract.

Theorems 1.2 and 1.3 are proved in the next section. Surprisingly enough, our construction for the proof of Theorem 1.2 really uses the $\ell_{1}$-norm, and we are unable to adapt it to the Hilbertian setting. Thus the following question is still open:

QUeSTION 1.4. Let $\varepsilon \in(0,1)$ and suppose that $T \in \mathcal{B}(H)$ is an $\varepsilon$-hypercyclic operator acting on a Hilbert space $H$. Must $T$ be hypercyclic?

\section{Proof of Theorem 1.2}

2.1. Outline of the proof of Theorem 1.2. Fix $\varepsilon \in(0,1)$ and a positive integer $a$ such that $\varepsilon>2^{-a+1}$. Let $X$ be the space $\ell_{1}(\mathbb{N})$ endowed with the canonical basis $\left(e_{n}\right)_{n \geq 0}$. Our operator $T$ will act on the $\ell_{1}$-direct sum $Y=\bigoplus_{i=0}^{\infty} X$ of countably many copies of $\ell_{1}(\mathbb{N})$.

Let $\left(y^{(k)}\right)_{k \geq 1}$ be a sequence of vectors of $Y$ which has the following properties:

(i) the set $\left\{y^{(k)} ; k \geq 1\right\}$ is dense in $Y$;

(ii) each $y^{(k)} \in Y$ can be written as a sequence $y^{(k)}=\left(y_{1}^{(k)}, \ldots, y_{k-1}^{(k)}, 0, \ldots\right)$, where each $y_{j}^{(k)}$ is a vector of $X=\ell_{1}(\mathbb{N})$ which is in the linear span of the vectors $e_{i}, i \leq k-1$;

(iii) $2^{-k} \leq\left\|y_{j}^{(k)}\right\|$ for every $j=0, \ldots, k-1$, and $\left\|y^{(k)}\right\| \leq \frac{2^{k}}{1+2^{-a}}$.

For each $k \geq 1$ and each $j \leq k-1$, define $z_{j}^{(k)}=y_{j}^{(k)}+2^{-a}\left\|y_{j}^{(k)}\right\| e_{k^{2}+j}$ : it is a perturbation of the vector $y_{j}^{(k)}$ obtained by adding to it a (not too small) multiple of the basis vector $e_{k^{2}+j}$, which is far away from the support of $y_{j}^{(k)}$. We have $2^{-k} \leq\left\|z_{j}^{(k)}\right\|$ for each $j \leq k-1$. We then define $z^{(k)} \in Y$ by $z^{(k)}=\left(z_{0}^{(k)}, \ldots, z_{k-1}^{(k)}, 0, \ldots\right)$. Clearly $\left\|z^{(k)}\right\| \leq 2^{k}$.

Set $n_{0}=n_{0}^{\prime}=0$. Our goal is to construct by induction a sequence $\left(S_{j}\right)_{j \geq 1}$ of bounded operators on $X$ and two strictly increasing sequences of positive integers $\left(n_{k}\right)_{k \geq 1}$ and $\left(n_{k}^{\prime}\right)_{k \geq 1}$ such that $n_{k-1}^{\prime} \leq n_{k-1}^{\prime}+n_{k-1} \leq n_{k}<n_{k}+k<n_{k}^{\prime}$ for every $k \in \mathbb{N}$ and the six following properties hold true:

(a) each operator $S_{j}$ is bounded and invertible with $\left\|S_{j}^{-1}\right\| \leq 2$;

(b) $S_{j} e_{0}=e_{0}$ for every $j \in \mathbb{N}$;

(c) $\left\|S_{j} S_{j-1} \ldots S_{1}\right\| \leq 2^{a+1}$ for every $j \in \mathbb{N}$;

(d) $S_{n_{k}^{\prime}} \ldots S_{2} S_{1}=I$ (the identity operator) for every $k \in \mathbb{N}$;

(e) $S_{j}=I$ for every $k \in \mathbb{N}$ and every $j$ such that $n_{k}-n_{k-1}<j \leq n_{k}+k$ );

(f) $\left\|S_{n_{k}} \cdots S_{j} S_{j+1} z_{j}^{(k)}\right\| \leq 2^{-2 k-a}$ for every $k \in \mathbb{N}$ and every $j=0, \ldots, k-1$.

Suppose that $\left(n_{k}\right),\left(n_{k}^{\prime}\right)$ and $\left(S_{j}\right)$ have been constructed so as to satisfy properties (a) to (f). Consider on $Y$ the operator $T$ which is the backward shift with operator- 
weights $S_{j}^{-1}$ : for any sequence $\left(v_{j}\right)_{j \geq 0}$ of $Y$,

$$
T\left(v_{0}, v_{1}, \ldots\right)=\left(S_{1}^{-1} v_{1}, S_{2}^{-1} v_{2}, \ldots\right) .
$$

Clearly $T$ is bounded on $Y$ with $\|T\| \leq 2$ by (a). For any $n \in \mathbb{N}$ we have

$$
T^{n}\left(v_{0}, v_{1}, \ldots\right)=\left(S_{1}^{-1} \ldots S_{n}^{-1} v_{n}, S_{2}^{-1} \ldots S_{n+1}^{-1} v_{n+1}, \ldots, S_{j+1}^{-1} \ldots S_{n+j}^{-1} v_{n+j}, \ldots\right) .
$$

For $k \in \mathbb{N}$ define $x^{(k)} \in Y$ by

$$
x^{(k)}=(\underbrace{0, \ldots, 0}_{n_{k}}, S_{n_{k}} \ldots S_{1} z_{0}^{(k)}, S_{n_{k}} \ldots S_{2} z_{1}^{(k)}, \ldots, S_{n_{k}} \ldots S_{k} z_{k-1}^{(k)}, 0, \ldots) .
$$

By (f), we have $\left\|x^{(k)}\right\| \leq 2^{-2 k-a}\left\|z^{(k)}\right\| \leq 2^{-k-a}$, and thus the vector

$$
x=\sum_{k=1}^{\infty} x^{(k)}
$$

belongs to $Y$.

2.2. The vector $x$ is $\varepsilon$-hypercyclic for $T$. Let $k \in \mathbb{N}$. Observe that by (e) we can rewrite $x^{(k)}$ as

$$
x^{(k)}=(\underbrace{0, \ldots, 0}_{n_{k}}, S_{n_{k}} \ldots S_{1} z_{0}^{(k)}, S_{n_{k}+1} \ldots S_{2} z_{1}^{(k)}, \ldots, S_{n_{k}+k-1} \ldots S_{k} z_{k-1}^{(k)}, 0, \ldots),
$$

and so $T^{n_{k}} x^{(k)}=z^{(k)}$. Clearly $T^{n_{k}} x^{(m)}=0$ for $m<k$, and for $m>k$ we have $T^{n_{k}} x^{(m)}=x^{(m)}$ by (e) again. Hence

$$
\left\|T^{n_{k}} x-z^{(k)}\right\|=\left\|\left(\sum_{m=k+1}^{\infty} x^{(m)}\right)\right\| \leq \sum_{m=k+1}^{\infty} 2^{-m-a}=2^{-k-a} .
$$

Let $v$ be any non zero vector of $Y$. Choose $k \in \mathbb{N}$ such that $\left\|v-y^{(k)}\right\|<\varepsilon^{\prime}\|v\|$, where $\varepsilon^{\prime}>0$ satisfies $\left(1+\varepsilon^{\prime}\right) 2^{1-a}+\varepsilon^{\prime}<\varepsilon$. Then $\left\|y^{(k)}\right\|<\|v\|\left(1+\varepsilon^{\prime}\right)$ and

$$
\begin{aligned}
\left\|T^{n_{k}} x-v\right\| & \leq\left\|T^{n_{k}} x-z^{(k)}\right\|+\left\|z^{(k)}-y^{(k)}\right\|+\left\|y^{(k)}-v\right\| \\
& \leq 2^{-k-a}+2^{-a}\left\|y^{(k)}\right\|+\varepsilon^{\prime}\|v\| \leq\left\|y^{(k)}\right\| 2^{-a+1}+\varepsilon^{\prime}\|v\| \\
& \leq\|v\|\left(\left(1+\varepsilon^{\prime}\right) 2^{-a+1}+\varepsilon^{\prime}\right) \leq \varepsilon\|v\| .
\end{aligned}
$$

Hence $x$ is an $\varepsilon$-hypercyclic vector for $T$.

2.3. The operator $T$ is not hypercyclic on $Y$. Suppose on the contrary that there is a vector $v=\left(v_{0}, v_{1}, \ldots\right) \in Y$ hypercyclic for $T$. Then there exists an increasing sequence $\left(m_{j}\right)_{j \geq 0}$ of integers such that the quantity $\left\|T^{m_{j}} v-\left(e_{0}, 0, \ldots\right)\right\|$ tends to zero as $j$ tends to infinity. In particular, reading this on the first coordinate yields that $\left\|S_{1}^{-1} S_{2}^{-1} \ldots S_{m_{j}}^{-1} v_{m_{j}}-e_{0}\right\|$ tends to zero. Here assumptions (b) and (c) come into play:

$$
\begin{aligned}
\left\|v_{m_{j}}-e_{0}\right\| & =\left\|v_{m_{j}}-S_{m_{j}} \cdots S_{1} e_{0}\right\| \leq\left\|S_{m_{j}} \cdots S_{1}\right\| \cdot\left\|S_{1}^{-1} \cdots S_{m_{j}}^{-1} v_{m_{j}}-e_{0}\right\| \\
& \leq 2^{a+1}\left\|S_{1}^{-1} \cdots S_{m_{j}}^{-1} v_{m_{j}}-e_{0}\right\|
\end{aligned}
$$

Hence $\left\|v_{m_{j}}-e_{0}\right\|$ tends to zero, thus $\left\|v_{m_{j}}\right\|$ tends to 1 , which contradicts the assumption that $v$ belongs to $Y$. 
2.4. Construction of the sequences $\left(n_{k}\right)_{k \geq 0},\left(n_{k}^{\prime}\right)_{k \geq 0}$ and $\left(S_{j}\right)_{j \geq 1}$. Recall that we set formally $n_{0}=n_{0}^{\prime}=0$. Define the numbers $n_{k}, n_{k}^{\prime}$ inductively by setting

$$
n_{k}=n_{k-1}^{\prime}+4 k+2 a+1+n_{k-1}
$$

and

$$
n_{k}^{\prime}=n_{k}+5 k+2 a+1
$$

We define the operators $S_{j}$ by induction: at step $k$ the operators $S_{j}$ are constructed for $n_{k-1}^{\prime}<j \leq n_{k}^{\prime}$. So let $k \geq 1$ and suppose that $S_{j} \in B(X)$ are already defined and invertible for $j \leq n_{k-1}^{\prime}$. For $0 \leq i \leq k-1$ write

$$
w_{i}^{(k)}=S_{1}^{-1} \cdots S_{i}^{-1} y_{i}^{(k)}
$$

and

$$
\alpha_{i}^{(k)}=2^{-a}\left\|y_{i}^{(k)}\right\| \cdot\left\|S_{1}^{-1} \cdots S_{i}^{-1} e_{k^{2}+i}\right\|
$$

Note that for $k=1$ we have $w_{0}^{(1)}=y_{0}^{(1)}$ and $\alpha_{0}^{(1)}=2^{-a}\left\|y_{0}^{(1)}\right\|$.

At step $k \geq 2$ we have already defined in particular the invertible operators $S_{1}, \ldots, S_{k-1}$, since $k-1 \leq n_{k-1}^{\prime}$.

We define the operators $S_{j}, n_{k-1}^{\prime}<j \leq n_{k}^{\prime}$, by defining $S_{j} e_{i}$, depending on the values of $i$ and $j$ :

- For $i<k^{2}$, define

(1) $S_{j} e_{i}=e_{i} \quad$ for $n_{k-1}^{\prime}<j \leq n_{k}^{\prime}$.

- For $k^{2} \leq i \leq k^{2}+k-1$, define

(2a) $S_{j} e_{i}=2 e_{i} \quad\left(n_{k-1}^{\prime}<j \leq n_{k-1}^{\prime}+a\right)$;

(2b) $S_{j} e_{i}=-\frac{w_{i-k^{2}}^{(k)}}{2^{a} \alpha_{i-k^{2}}^{(k)}}+e_{i} \quad\left(j=n_{k-1}^{\prime}+a+1\right)$;

(2c) $S_{j} e_{i}=\frac{1}{2} e_{i} \quad\left(n_{k-1}^{\prime}+a+1<j<n_{k-1}^{\prime}+2 a+4 k+1=n_{k}-n_{k-1}\right)$;

(2d) $S_{j} e_{i}=e_{i} \quad\left(n_{k}-n_{k-1}<j \leq n_{k}+k\right)$;

(2e) $S_{j} e_{i}=2 e_{i} \quad\left(n_{k}+k<j \leq n_{k}+5 k+a\right)$;

(2f) $S_{j} e_{i}=\frac{w_{i-k^{2}}^{(k)}}{2^{a} \alpha_{i-k^{2}}^{(k)}}+e_{i} \quad\left(j=n_{k}+5 k+a+1\right)$;

$(2 \mathrm{~g}) S_{j} e_{i}=\frac{1}{2} e_{i} \quad\left(n_{k}+5 k+a+1<j \leq n_{k}+5 k+2 a+1=n_{k}^{\prime}\right)$.

- For $i>k^{2}+k-1$, define

(3a) $S_{j} e_{i}=\frac{1}{2} e_{i} \quad\left(n_{k-1}^{\prime}<j \leq n_{k}-n_{k-1}\right)$;

(3b) $S_{j} e_{i}=e_{i} \quad\left(n_{k}-n_{k-1}<j \leq n_{k}+k\right)$;

(3c) $S_{j} e_{i}=\frac{1}{2} e_{i} \quad\left(n_{k}+k<j \leq n_{k}^{\prime}-1\right)$;

(3d) $S_{j} e_{i}=2^{n_{k}^{\prime}-n_{k-1}^{\prime}-n_{k-1}-k-1} e_{i} \quad\left(j=n_{k}^{\prime}\right)$.

For $k \in \mathbb{N}$ let $M_{k}=\overline{\mathrm{sp}}\left[e_{i} ; i=0 \ldots k^{2}+k-1\right]$ and $L_{k}=\overline{\mathrm{sp}}\left[e_{i} ; i>k^{2}+k-1\right]$.

2.5. Boundedness and invertibility of the operators $S_{j}$. We show first by induction on $k$ that the operators $S_{j}, n_{k-1}^{\prime}<j \leq n_{k}^{\prime}$, defined above are bounded, invertible and upper triangular, and that their inverses $S_{j}^{-1}$ are also bounded and upper triangular.

Prepared using etds.cls 
As mentioned above, for $k=1$ we have $w_{0}^{(1)}=y_{0}^{(1)} \in \mathbb{C} \cdot e_{0}$, so the operators $S_{j}, j \leq n_{1}^{\prime}$ are upper triangular. Moreover, for each $j \leq n_{1}^{\prime}$ we have $S_{j}\left(M_{1}\right) \subseteq M_{1}$, $S_{j}\left(L_{1}\right) \subseteq L_{1}$. The operator $S_{j} \mid M_{1}$ is upper triangular with a positive main diagonal and $S_{j} \mid L_{1}$ is a nonzero scalar multiple of the identity operator. So $S_{j}$ is bounded and invertible and its inverse $S_{j}^{-1}$ is also bounded and upper triangular.

Suppose that $k \geq 2$ and that the operators $S_{j}, S_{j}^{-1}, j \leq n_{k-1}^{\prime}$, are bounded, invertible and upper triangular. For $0 \leq i \leq k-1, y_{i}^{(k)}$ belongs to the linear span of the vectors $e_{l}, l=0 \ldots k-1$, and so this is also the case for the vector $w_{i}^{(k)}=S_{1}^{-1} \cdots S_{k-1}^{-1} y_{i}^{(k)}$. Hence the operators $S_{j}, n_{k-1}^{\prime}<j \leq n_{k}^{\prime}$ defined by $(1)-$ (3) are upper triangular. As above, we conclude that they are also bounded and invertible, and that their inverses $S_{j}^{-1}$ are also bounded and upper triangular.

We now have to show that the operators $S_{j}$ satisfy conditions (a)-(f).

2.6. Proof of properties (b), (e) and (d). By definition, $S_{j} e_{0}=e_{0}$ for all $j$ and $S_{j}$ is equal to the identity operator for $n_{k}-n_{k-1}<j \leq n_{k}+k$. Hence conditions (b) and (e) are satisfied trivially. Then we have to prove by induction on $k$ that $S_{n_{k}^{\prime}} \cdots S_{1}=I$, i.e., that $S_{n_{k}^{\prime}} \cdots S_{n_{k-1}^{\prime}+1}=I$ :

- for $i<k^{2}$, clearly $S_{n_{k}^{\prime}} \cdots S_{n_{k-1}^{\prime}+1} e_{i}=e_{i}$ since all the operators $S_{j}, n_{k-1}^{\prime}+1 \leq$ $j \leq n_{k}^{\prime}$, act on $e_{i}$ as the identity operator by (1);

- for $i>k^{2}+k-1$ it is also easy to check using property (3) that $S_{n_{k}^{\prime}} \cdots S_{n_{k-1}^{\prime}+1} e_{i}=e_{i}$ (just multiply all coefficients together);

- for $k^{2} \leq i \leq k^{2}+k-1$ we have that $S_{n_{k}^{\prime}} \cdots S_{n_{k-1}^{\prime}+1} e_{i}$ is equal to

$$
S_{n_{k}^{\prime}} \cdots S_{n_{k-1}^{\prime}+a+1}\left(2^{a} e_{i}\right)=S_{n_{k}^{\prime}} \cdots S_{n_{k-1}^{\prime}+a+2}\left(-\frac{w_{i-k^{2}}^{(k)}}{\alpha_{i-k^{2}}^{(k)}}+2^{a} e_{i}\right)
$$

by (2b). Then since $w_{i-k^{2}}^{(k)} / \alpha_{i-k^{2}}^{(k)}$ is supported by the first $k$ vectors $e_{l}, l=$ $0, \ldots, k-1$, by $(2 \mathrm{c}),(2 \mathrm{~d})$ and $(2 \mathrm{e})$ applied successively this quantity is equal to

$$
S_{n_{k}^{\prime}} \cdots S_{n_{k-1}^{\prime}+2 a+4 k+2}\left(-\frac{w_{i-k^{2}}^{(k)}}{\alpha_{i-k^{2}}^{(k)}}+2^{-4 k} e_{i}\right)
$$

and so equal to

$$
S_{n_{k}^{\prime}} \cdots S_{n_{k}+k+1}\left(-\frac{w_{i-k^{2}}^{(k)}}{\alpha_{i-k^{2}}^{(k)}}+2^{-4 k} e_{i}\right)=S_{n_{k}^{\prime}} \cdots S_{n_{k}+5 k+a+1}\left(-\frac{w_{i-k^{2}}^{(k)}}{\alpha_{i-k^{2}}^{(k)}}+2^{a} e_{i}\right) .
$$

Then the expression in (2f) destroys the quantity $w_{i-k^{2}}^{(k)} / \alpha_{i-k^{2}}^{(k)}$ in this expression, and we eventually get that

$$
S_{n_{k}^{\prime}} \cdots S_{n_{k-1}^{\prime}+2 a+4 k+2}\left(-\frac{w_{i-k^{2}}^{(k)}}{\alpha_{i-k^{2}}^{(k)}}+2^{-4 k} e_{i}\right)=S_{n_{k}^{\prime}} \cdots S_{n_{k}+5 k+a+2}\left(2^{a} e_{i}\right)=e_{i} .
$$

Hence $S_{n_{k}^{\prime}} \cdots S_{n_{k-1}^{\prime}+1}=I$ and property (d) is proved. 
2.7. Proof of property (a). We now have prove by induction on $k$ that $\left\|S_{j}^{-1}\right\| \leq 2$ for every $j$ with $n_{k-1}^{\prime}<j \leq n_{k}^{\prime}$. Let $k \geq 1$ and suppose that $\left\|S_{j}^{-1}\right\| \leq 2$ for every $j \leq n_{k-1}^{\prime}$. For $0 \leq i \leq k-1$ we have

$$
S_{1}^{-1} \cdots S_{i}^{-1} e_{k^{2}+i}=\left\|S_{1}^{-1} \cdots S_{i}^{-1} e_{k^{2}+i}\right\| \cdot e_{k^{2}+i}
$$

since the operators $S_{1}^{-1}, \ldots, S_{i}^{-1}$ just multiply the vector $e_{k^{2}+i}$ by some coefficient. Thus

$S_{1}^{-1} \cdots S_{i}^{-1} z_{i}^{(k)}=S_{1}^{-1} \cdots S_{i}^{-1} y_{i}^{(k)}+2^{-a}\left\|y_{i}^{(k)}\right\| S_{1}^{-1} \cdots S_{i}^{-1} e_{k^{2}+i}=w_{i}^{(k)}+\alpha_{i}^{(k)} e_{k^{2}+i}$.

Let $r=\operatorname{card}\left\{s ; 1 \leq s \leq i\right.$ and $\left.S_{s} \neq I\right\}$. Then $\left\|S_{i}^{-1} \cdots S_{i}^{-1} y_{i}^{(k)}\right\| \leq 2^{r} \cdot\left\|y_{i}^{(k)}\right\|$ by the induction assumption and $\left\|S_{1}^{-1} \cdots S_{i}^{-1} e_{k^{2}+i}\right\|=2^{r}$ by (3a). Hence

$$
\alpha_{i}^{(k)}=2^{-a}\left\|y_{i}^{(k)}\right\| 2^{r} \geq 2^{-a}\left\|S_{i}^{-1} \cdots S_{i}^{-1} y_{i}^{(k)}\right\| \geq 2^{-a}\left\|w_{i}^{(k)}\right\| .
$$

Clearly $\left\|S_{j}^{-1}\right\| \leq 2$ for all $j$ with $n_{k-1}^{\prime}<j \leq n_{k}^{\prime}, j \neq n_{k-1}^{\prime}+a+1$ and $j \neq n_{k}+5 k+a+1$. In order to prove that $\left\|S_{j}^{-1}\right\| \leq 2$ in these two cases, we only have to check that $\left\|S_{j}^{-1} e_{i}\right\| \leq 2$ for every $i \geq 0$ : observe that at this point we use the $\ell_{1}$-norm in a crucial way.

- If $i<k^{2}$ then , $\left\|S_{n_{k-1}^{\prime}+a+1}^{-1} e_{i}\right\|=\left\|e_{i}\right\| \leq 2$ and $\left\|S_{n_{k}+5 k+a+1}^{-1} e_{i}\right\| \leq 2$ by (1).

- Similarly, if $i>k^{2}+k-1$ then $\left\|S_{n_{k-1}^{\prime}+a+1}^{-1} e_{i}\right\| \leq 2$ and $\left\|S_{n_{k}+5 k+a+1}^{-1} e_{i}\right\| \leq 2$ by $(3)$.

- Let $k^{2} \leq i \leq k^{2}+k-1$. Then

$$
S_{n_{k-1}^{\prime}+a+1} S_{n_{k}+5 k+a+1} e_{i} S_{n_{k}+5 k+a+1} S_{n_{k-1}^{\prime}+a+1} e_{i}=e_{i} .
$$

So $\left\|S_{n_{k}+5 k+a+1}^{-1} e_{i}\right\|=\left\|S_{n_{k-1}^{\prime}+a+1} e_{i}\right\| \leq 1$ and $\left\|S_{n_{k-1}^{\prime}+a+1}^{-1} e_{i}\right\|=\left\|S_{n_{k}+5 k+a+1} e_{i}\right\| \leq$ 2 .

This proves (a).

2.8. Proof of property $(f)$. Let $k \in \mathbb{N}$ and $0 \leq i \leq k-1$. Then

$$
\begin{aligned}
\left\|S_{n_{k}} \cdots S_{i+1} z_{i}^{(k)}\right\| & =\left\|S_{n_{k}} \cdots S_{1}\left(S_{1}^{-1} \cdots S_{i}^{-1}\right) z_{i}^{(k)}\right\| \\
& =\left\|S_{n_{k}} \cdots S_{n_{1}}\left(w_{i}^{(k)}+\alpha_{i}^{(k)} e_{k^{2}+i}\right)\right\| \\
& =\left\|S_{n_{k}} \cdots S_{n_{k-1}^{\prime}+1}\left(w_{i}^{(k)}+\alpha_{i}^{(k)} e_{k^{2}+i}\right)\right\| \\
& =\left\|S_{n_{k}} \cdots S_{n_{k-1}^{\prime}+a+1}\left(w_{i}^{(k)}+2^{a} \alpha_{i}^{(k)} e_{k^{2}+i}\right)\right\| \\
& =\left\|S_{n_{k}} \cdots S_{n_{k-1}^{\prime}+a+2}\left(2^{a} \alpha_{i}^{(k)} e_{k^{2}+i}\right)\right\| \\
& =\left\|S_{n_{k}} \cdots S_{n_{k-1}^{\prime}+2 a+4 k+2}\left(2^{-4 k} \alpha_{i}^{(k)} e_{k^{2}+i}\right)\right\| \\
& =\left\|2^{-4 k} \alpha_{i}^{(k)} e_{k^{2}+i}\right\| \\
& =2^{-4 k} \alpha_{i}^{(k)} .
\end{aligned}
$$

Then (f) is proved by observing that

$$
2^{-4 k} \alpha_{i}^{(k)}=2^{-4 k} 2^{-a}\left\|y_{i}^{(k)}\right\| \cdot\left\|S_{1}^{-1} \cdots S_{i}^{-1} e_{k^{2}+i}\right\| \leq 2^{-4 k} 2^{-a} 2^{k} \cdot 2^{k}=2^{-2 k-a} .
$$


2.9. Proof of property (c). It remains to show that $\left\|S_{j} \cdots S_{1}\right\| \leq 2^{a+1}$ for all $j \in \mathbb{N}$. By $(\mathrm{d})$, it is sufficient to show that $\left\|S_{j} \cdots S_{n_{k-1}^{\prime}}\right\| \leq 2^{a+1}$ for all $k \in \mathbb{N}$ and $n_{k-1}^{\prime}<j \leq n_{k}^{\prime}$. Equivalently, using again the $\ell_{1}$-norm, it must be proved that $\left\|S_{j} \cdots S_{n_{k-1}^{\prime}+1} e_{i}\right\| \leq 2^{a+1}$ for every $i \geq 0$ and $n_{k-1}^{\prime}<j \leq n_{k}^{\prime}$.

- For $i<k^{2}$ this is clear since the operators $S_{j}, n_{k-1}^{\prime}<j \leq n_{k}^{\prime}$, act on $e_{i}$ as the identity operator.

- For $i>k^{2}+k-1$ this is also clear: $\left\|S_{j} \cdots S_{n_{k-1}^{\prime}+1} e_{i}\right\| \leq 1$ for all $j$, $n_{k-1}^{\prime}+1 \leq j \leq n_{k}^{\prime}$ (just multiply the coefficients, the worst case being when $j=n_{k}^{\prime}$ ).

- For $k^{2} \leq i \leq k^{2}+k-1$, the sequence

$$
S_{n_{k-1}^{\prime}+1} e_{i}, S_{n_{k-1}^{\prime}+2} S_{n_{k-1}^{\prime}+1} e_{i}, \ldots, S_{n_{k}^{\prime}} \cdots S_{n_{k-1}^{\prime}+1} e_{i}
$$

is equal to $2 e_{i}, \ldots, 2^{a} e_{i},-\frac{w_{i-k^{2}}^{(k)}}{\alpha_{i-k^{2}}^{(k)}}+2^{a} e_{i}, \ldots,-\frac{w_{i-k^{2}}^{(k)}}{\alpha_{i-k^{2}}^{(k)}}+2^{-4 k} e_{i}, \ldots,-\frac{w_{i-k^{2}}^{(k)}}{\alpha_{i-k^{2}}^{(k)}}+$ $2^{-4 k} e_{i},-\frac{w_{i-k^{2}}^{(k)}}{\alpha_{i-k^{2}}^{(k)}}+2^{a} e_{i}, 2^{a} e_{i}, \ldots, e_{i}$. Hence

$$
\max _{n_{k-1}^{\prime}+1 \leq j \leq n_{k}^{\prime}}\left\|S_{j} \cdots S_{n_{k-1}^{\prime}+1} e_{i}\right\|=\left\|-\frac{w_{i-k^{2}}^{(k)}}{\alpha_{i-k^{2}}^{(k)}}+2^{a} e_{i}\right\|=\left|\frac{w_{i-k^{2}}^{(k)}}{\alpha_{i-k^{2}}^{(k)}}\right|+2^{a} \leq 2^{a+1} .
$$

This proves (c).

Thus the operators $S_{j}, j \in \mathbb{N}$, satisfy all the properties (a) to (f), and consequently the operator $T$ defined here is $\varepsilon$-hypercyclic but not hypercyclic on $Y=\bigoplus_{\ell_{1}} \ell_{1}(\mathbb{N})$. This finishes the proof of Theorem 1.2.

2.10. Possible extensions of the method. In the same way it is possible to construct a non-hypercyclic operator $T$ on $\ell_{1}(\mathbb{N})$ on an infinite-dimensional separable Hilbert space such that $T \oplus T$ is $\varepsilon$-hypercyclic. Indeed, consider the space $Y$ as in Theorem 1.2 and a sequence of pairs of vectors $\left(y^{(k)}, \widetilde{y}^{(k)}\right)$ which is dense in $Y \oplus Y$. In the same way one can construct a vector $x \oplus \widetilde{x} \in Y \oplus Y$ which is $\varepsilon$-hypercyclic for $T \oplus T$. One can even have that $T_{n}=\underbrace{T \oplus \cdots \oplus T}_{n}$ is $\varepsilon$-hypercyclic for each $n \in \mathbb{N}$. Details are left to the reader.

\section{Proof of Theorem 1.3}

3.1. Point spectrum of the adjoint of an $\varepsilon$-hypercyclic operator. We need the following auxiliary result:

Lemma 3.1. Let $0<\varepsilon<1$ and let $T \in B(X)$ be an $\varepsilon$-hypercyclic operator. Then the point spectrum $\sigma_{p}\left(T^{*}\right)$ of the adjoint of $T$ is empty.

Proof. Suppose on the contrary that $\alpha$ belongs to $\sigma_{p}\left(T^{*}\right)$. Let $y^{*} \in X^{*}$ satisfy $\left\|y^{*}\right\|=1$ and $T^{*} y^{*}=\alpha y^{*}$, and let $x \in X$ be an $\varepsilon$-hypercyclic vector for $T$. We distinguish two cases. 
- First case. We have either $\left\langle x, y^{*}\right\rangle=0$, or $|\alpha| \leq 1$. Choose $t>(\|x\|+1) /(1-\varepsilon)$ and $y \in X$ with $\|y\|=1$ and $\left\langle y, y^{*}\right\rangle>1-\varepsilon / t$. Since $x$ is an $\varepsilon$-hypercyclic vector for $T$, there exists an $n \geq 0$ such that $\left\|T^{n} x-t y\right\| \leq \varepsilon\|t y\|=t \varepsilon$. So $\left|\left\langle T^{n} x-y, y^{*}\right\rangle\right| \leq t \varepsilon$. On the other hand,

$$
\left|\left\langle T^{n} x-t y, y^{*}\right\rangle\right| \geq\left|\left\langle t y, y^{*}\right\rangle\right|-\left|\left\langle T^{n} x, y^{*}\right\rangle\right| \geq t-\varepsilon-|\alpha|^{n}\left|\left\langle x, y^{*}\right\rangle\right| \geq t-1-\|x\| .
$$

Thus $t-1-\|x\| \leq t \varepsilon$ and so $t \leq(1+\|x\|) /(1-\varepsilon)$, a contradiction.

- Second case. We have $\left\langle x, y^{*}\right\rangle \neq 0$ and $|\alpha|>1$. Choose $y \in X$ such that $0 \neq\|y\|<\left|\left\langle x, y^{*}\right\rangle\right| /(1+\varepsilon)$. There exists $n \geq 0$ such that $\left\|T^{n} x-y\right\| \leq \varepsilon\|y\|$, and thus $\left|\left\langle T^{n} x-y, y^{*}\right\rangle\right| \leq \varepsilon\|y\|$. On the other hand,

$$
\left|\left\langle T^{n} x-y, y^{*}\right\rangle\right| \geq\left|\left\langle T^{n} x, y^{*}\right\rangle\right|-\left|\left\langle y, y^{*}\right\rangle\right| \geq\left|\alpha^{n}\right| \cdot\left|\left\langle x, y^{*}\right\rangle\right|-\|y\|>\left|\left\langle x, y^{*}\right\rangle\right|-\|y\| .
$$

Thus $\left|\left\langle x, y^{*}\right\rangle\right|-\|y\|<\varepsilon\|y\|$, and so $\|y\|>\left(\left|\left\langle x, y^{*}\right\rangle\right|\right)(1+\varepsilon)$, a contradiction again.

3.2. Proof of Theorem 1.3. Lemma 3.1 shows that we can assume that $X$ is infinite dimensional. We are going to prove that $T$ is topologically transitive, i.e. that for every nonempty open subsets $U$ and $V$ of $X$ there exists an integer $n \in \mathbb{N}$ such that $T^{n}(U) \cap V$ is nonempty. Let $u \in U$ and $v \in V$ be two nonzero vectors of $U$ and $V$ respectively, and let $\delta>0$ be so small that $B(u, \delta) \subseteq U, B(v, \delta) \subseteq V$ and $\delta<\min (\|u\|,\|v\|)$. Let $x \in X$ be an $\varepsilon$-hypercyclic vector for $T$, where $\varepsilon<\delta /(6 \max (\|u\|,\|v\|))$. There exists $n_{0} \geq 0$ such that $\left\|T^{n_{0}} x-u\right\| \leq \varepsilon\|u\|<\delta$, and so $T^{n_{0}} x$ belongs to $U$. Let us now show that there exist infinitely many $n$ 's such that $T^{n} x$ belongs to $V$. Suppose on the contrary that there are only finitely many such integers $n_{1}, \ldots, n_{k}$. As above, for each $v^{\prime} \in X$ with $\left\|v^{\prime}-v\right\|<\frac{2 \delta}{3}$ there exists an integer $n\left(v^{\prime}\right)$ which satisfies $\left\|T^{n\left(v^{\prime}\right)} x-v^{\prime}\right\| \leq \varepsilon\left\|v^{\prime}\right\| \leq 2 \varepsilon\|v\|<\delta / 3$. Since $\| T^{n\left(v^{\prime}\right)} x-$ $v\|\leq\| T^{n\left(v^{\prime}\right)} x-v^{\prime}\|+\| v^{\prime}-v \|<\delta$, we have $n\left(v^{\prime}\right) \in\left\{n_{1}, \ldots, n_{k}\right\}$ and the ball $B(v,(2 \delta) / 3)$ is covered by a finite number of balls $B\left(T^{n_{1}} x, \delta / 3\right), \ldots, B\left(T^{n_{k}} x, \delta / 3\right)$. However, in an infinite dimensional space this is not possible. Hence there are infinitely many $n$ 's with $\left\|T^{n} x-v\right\|<\delta$, and in particular, there exists $n_{1}>n_{0}$ such that $T^{n_{1}} x$ is in $V$. So $T^{n_{1}-n_{0}} T^{n_{0}} x=T^{n_{1}} x \in V \cap T^{n_{1}-n_{0}}(U)$, and consequently $T$ is hypercyclic.

\section{REFERENCES}

[1] F. Bayart, É. Matheron, Dynamics of linear operators, book to appear, Cambridge University Press.

[2] F. BAyArt, É. Matheron, (Non)-weakly mixing operators and hypercyclicity sets, preprint 2007.

[3] P. Bourdon, N. Feldman, Somewhere dense orbits are everywhere dense, Indiana Univ. Math. J., 52 (2003), pp $811-819$.

[4] K. Chan, R. SAnders, A weakly hypercyclic operator that is not norm hypercyclic, J. Operator Th. 52 (2004), pp $39-59$. 
[5] G. Costakis, On a conjecture of D. Herrero concerning hypercyclic operators, $C . R$. Acad. Sci. Paris Ser. I Math., 330 (2000), pp $179-182$.

[6] N. Feldman, Perturbations of hypercyclic vectors, J. Math. Anal. Appl., 273 (2002), pp $67-74$.

[7] K.-G. Grosse-Erdmann, A. Peris, Frequently dense orbits, C. R. Math. Acad. Sci. Paris, 341 (2005), pp $123-128$.

[8] V. MülLER, Three problems, Mini-Workshop Hypercyclicity and Linear Chaos, organized by T. Bermudez, G. Godefroy, K.-G. Grosse-Erdmann and A. Peris, Oberwolfach Reports, 3 (2006), pp. $2227-2276$.

[9] A. Peris, Multi-hypercyclic operators are hypercyclic, Math. Z., 236 (2001), pp 779 -786 .

[10] S. Rolewicz, On orbits of elements, Studia Math., 32 (1969), pp $17-22$. 